\title{
Antidepressant use in clinical practice: efficacy $v$. effectiveness
}

\author{
JOHN DONOGHUE and TIMOTHY R. HYLAN
}

\begin{abstract}
Background Although the efficacy of antidepressants has been demonstrated in randomised, controlled clinical trials, it is how an antidepressant is used in clinical practice that determines its clinical effectiveness, or real-world efficacy.
\end{abstract}

Aims To explore the frequency with which antidepressants are used at adequate dose and duration to obtain remission of symptoms and prevent relapse in clinical practice and discuss potential implications for clinical outcomes.

Method Studies of antidepressant prescribing were reviewed and comparisons made between antidepressant classes and individual compounds within those classes.

Results Naturalistic studies show that patients who begin therapy on tricyclic antidepressants often receive sub-therapeutic doses for inadequate duration; conversely, patients who begin therapy on selective serotonin reuptake inhibitors more often receive an adequate dose of therapy for a longer duration.

Conclusions How antidepressants are used in clinical practice can determine the clinical outcomes that are achieved. Antidepressants that are more forgiving of sub-optimal prescribing and use patterns by providers and patients, respectively, may help to improve real-world efficacy.

\section{Declaration of interest}

J.D. received an honorarium and travel expenses from Eli Lilly \& Co. At the time of manuscript preparation, T.R.H. was employed at Eli Lilly \& Co.
The efficacy of antidepressants in the treatment of depression and other related affective disorders has been demonstrated in randomised controlled trials (RCTs) (Song et al, 1993; Anderson \& Tomenson, 1994; Hotopf et al, 1996; Geddes et al, 1999; Anderson, 2000). Taking an antidepressant drug at an effective dose for an adequate period reduces the number and severity of acute symptoms (Anderson \& Tomenson, 1994; Montejo et al, 1998), improves the patient's ability to function (Mintz et al, 1992; Sturm \& Wells, 1995) and minimises the risk of relapse or recurrence (Prien \& Kupfer, 1986; Maj et al, 1992; British Association for Psychopharmacology, 1993). In major depression, pooled data from meta-analyses of clinical trials have failed to show consistently that any one antidepressant or group of antidepressants has superior efficacy over any other (Anon., 1993; Song et al, 1993; Anderson \& Tomenson, 1994; Hotopf et al, 1996; Geddes et al, 1999; Anderson, 2000). Although one meta-analysis found tricyclic antidepressants (TCAs) to be more efficacious than selective serotonin reuptake inhibitors (SSRIs) in hospital in-patients (Anderson, 1998), another meta-analysis using different methodology failed to find any difference (Geddes et al, 1999).

However, the conditions in which clinical trials are conducted may be very different from the conditions of clinical practice. Clinical trials typically take place in highly selected populations treated under controlled and optimal conditions, with treatment determined by a clear study protocol. This type of study design is quite deliberate - to reduce the potential for bias and to ensure that any differences found can be attributed to differences in the properties of the compounds being investigated.

In contrast, treatment conditions in clinical practice are far removed from those of clinical trials, with no patient selection, no randomisation, and with variations in the behaviour of prescribers and patients and in the systems of health care in which treatment is delivered (Simon et al, 1995). Data suggest that patient compliance and adherence to medications in clinical practice may also differ from that in RCTs (Demyttenaere, 1997).

While the efficacy findings of RCTs are certainly important factors in antidepressant selection, the efficacy of a compound is no more than a measure of its therapeutic potential under standardised and optimal conditions. A more useful concept is that of real-world efficacy or 'effectiveness': the use of the compound in ways that will deliver the desired outcomes of treatment. Given the complexities of clinical practice, an antidepressant's efficacy as demonstrated by RCTs is likely to overestimate its effectiveness in clinical practice settings. Differences in effectiveness between antidepressant classes and individual compounds may help to guide antidepressant selection when efficacy is similar.

\section{TREATMENT GUIDELINES}

The findings of efficacy from clinical trials, and the conditions that need to be met to ensure effectiveness in clinical practice, have influenced the content of both national and international guidelines for the management of depression (Donoghue \& Taylor, 2000). Generally, guidelines recommend that to be effective antidepressants need to be used at a known effective dose, and to reduce the likelihood of relapse, treatment should be maintained at the same dose for a minimum period, usually 4-6 months following resolution of acute symptoms (Donoghue \& Taylor, 2000). In clinical practice there are many factors that may influence whether these conditions are met, including the pharmacological properties of the drug (half-life, dosing regimen); tolerability; patients' beliefs and behaviour (compliance); providers' beliefs and behaviour (experience, preferences); health care system characteristics (financing of health care benefits and ease of access to treatment); and societal factors (stigma of treatment, discrimination).

\section{Importance of dose}

The use of an efficacious dose is the first step in achieving a positive clinical outcome. Because dose and adverse effects are usually linked (i.e. the higher the dose, the greater the incidence and severity of adverse effects), in clinical practice physicians may seek to 
treat patients with as low a dose as possible (Wernicke et al, 1989; Dunner \& Dunbar, 1992; Fabre et al, 1995; Rudolph et al, 1998). However, treating patients with the lowest possible dose may subject them to adverse drug effects without the benefit of an efficacious drug treatment (Dunn et al, 1999). Compliance with the prescribed treatment is also an important factor in the patient receiving an appropriate dose (Johnson, 1986; Demyttenaere, 1997).

\section{Importance of duration}

Depression is a recurrent and chronic disorder. As many as $50 \%$ of patients who have an initial episode of depression will have a recurrent episode (Agency for Health Care Policy Research, 1993) and 12-15\% will become chronically depressed (Scott, 1988). Controlled studies have found that the initial duration of antidepressant therapy is an important determinant of relapse or recurrent episodes, with the risk of relapse decreasing as treatment time increases (Maj et al, 1992; Montgomery et al, 1992; Altamura \& Percudani, 1993). The importance of therapy length for symptom improvement in the acute phase (Montejo et al, 1998; Hylan et al, $1999 b$ ), restoration of patient functioning (Sturm \& Wells, 1995) and reduced risk of relapse or recurrence ( $\mathrm{Li}$ et al, 1998; Melfi et al, 1998) has also been confirmed in naturalistic studies. For example, one such study found that compared with 1 month of antidepressant treatment, 4 months of treatment increased measures of work restoration four-fold (Mintz et al, 1992). A second study found that doubling the proportion of patients receiving adequate antidepressant therapy resulted in a significant reduction in depression-related disabilities (Sturm \& Wells, 1995).

\section{Clinical practice realities}

A growing body of naturalistic research has contributed to our understanding of how antidepressants are used in clinical practice and whether such use fulfils the requirements of effectiveness (Donoghue \& Taylor, 2000). This research is especially valuable from the perspective of primary care, where the majority of cases of depression are treated (Paykel \& Priest, 1992). Evidence has been available since the early 1970 s that antidepressants are not used effectively in clinical practice (Johnson, 1973, 1981; Kotin et al, 1973; Keller et al, 1982). More recently, large population-based studies in several countries have confirmed, and indeed strengthened, the evidence from previous small studies that antidepressants are not being used effectively in primary care settings (Rosholm et al, 1993; Munizza et al, 1995; Bingefors et al, 1997). For physicians attempting to optimise treatment, these findings raise obvious questions of whether there are differences between antidepressants in how they are used in clinical practice and whether those differences are important in determining the likelihood of achieving effectiveness.

This review of the adequacy of antidepressant therapy in clinical practice focuses on TCAs and the SSRIs. Newer antidepressants - such as nefazodone, venlafaxine, mirtazapine and reboxetine - have only recently been introduced and few analyses of their use in clinical practice have been published.

\section{ADEQUACY OF DOSE}

\section{Tricyclic antidepressants}

In both primary and secondary care settings, TCAs are frequently prescribed at doses below those shown to be efficacious in RCTs, with a resultant decrease in effectiveness (Paykel et al, 1973; Berken et al, 1984; Garland et al, 1988; Rosholm et al, 1993; Henry et al, 1995; Beaumont et al, 1996; Donoghue \& Tylee, 1996; Donoghue et al, 1996; Katzelnick et al, 1996; Phillips et al, 1997; Donoghue \& Taylor, 2000). This may be due to a variety of factors, including concern about their side-effect and toxicity profiles, as well as continuing debate over their clinically effective dose (Thompson \& Thompson, 1989a; Beaumont et al, 1996; Canadian Coordinating Office, 1998; Donoghue, 1998a).

Low-dose prescribing of TCAs is not limited to a single geographical region, but has been found across Europe, North America and Australasia (Donoghue \& Taylor, 2000). For example, in 1993, Rosholm and colleagues found that in a Danish population of over 200000 patients, average doses of TCAs never reached $100 \mathrm{mg}$ daily (Rosholm et al, 1993). Similar findings have been made in Italy (Munizza et al, 1995) and in Sweden (Bingefors et al, 1997).

In the UK, a study using a national primary care database (DIN-Link, Compufile Ltd, Woking, UK) in a population of over 750000 patients revealed the all-pervasive extent of the use of TCAs at low doses, with
$88 \%$ of patients receiving doses below the minimum recommended in the national guidelines (Donoghue \& Tylee, 1996). This study was repeated comparing data on antidepressant use patterns from 1993 with data from 1995 - a period during which a national Defeat Depression campaign was being conducted (Donoghue et al, 1996). No differences were found in the doses of TCAs prescribed or the proportion of patients receiving an adequate dose. A further population-based study from Scotland, in a population of over 400000 patients, found that only $7 \%$ of prescriptions for TCAs reached therapeutic doses (MacDonald et al, 1996). However, these studies were limited by their cross-sectional design and by their inability to demonstrate outcomes of these patterns of treatment.

More recently, data have become available from the 5 years of the Defeat Depression campaign (further details available from the author upon request). There has been a slow trend for TCAs to be prescribed at effective doses in more patients, increasing from $12 \%$ in 1992 to $15 \%$ in 1996. However, for the most commonly prescribed TCAs, amitriptyline and dothiepin, the most commonly prescribed daily doses were $50 \mathrm{mg}$ and $75 \mathrm{mg}$ respectively, and these remained unchanged throughout the 5 years of the campaign. These doses are considerably less than the $125 \mathrm{mg}$ per day recommended as the minimum effective dose for these compounds (Paykel \& Priest, 1992). However, these cross-sectional findings may fail to reveal the full extent of the problem. Under this study design, a patient who was prescribed an effective dose for only one day would be included in the 'adequate dose' cohort.

Data published in 1998 demonstrated that these patterns of low-dose prescribing in the UK were not due to disproportionate numbers of elderly patients being treated with low doses; on the contrary, younger patients were more likely to receive an inadequate dose of TCA (Donoghue et al, 1998). The resultant decrease in effectiveness with low-dose prescribing has been demonstrated in studies by Blashki et al (1971) and Thompson \& Thompson (1989b), where patients treated with sub-optimal doses of amitriptyline or dothiepin had the same response as patients treated with placebo, and in a naturalistic study in primary care which found that three-quarters of patients treated with sub-therapeutic doses of antidepressants remained depressed despite long-term treatment (Ali, 1998). 


\section{SSRIs}

In contrast, SSRIs as a class are almost always prescribed at efficacious doses in the clinical practice setting, probably because of a combination of their better safety and toxicity profiles, tolerability and well-established simple dosing regimens (Donoghue \& Tylee, 1996; Donoghue et al, 1996; Katzelnick et al, 1996; Donoghue, 1998a,b; Montejo et al, 1998; Dunn et al, 1999). However, differences in dosing patterns also appear to exist between the SSRIs, although the clinical significance of these differences has not been established. Patients starting therapy with fluoxetine are less likely to have their dose increased above the initial dose than patients starting therapy with other SSRIs (Gregor et al, 1994; Navarro et al, 1995; Sclar et al, 1995; Donoghue \& Tylee, 1996; Truter \& Kotze, 1996; Bingefors et al, 1997; Donoghue, 1998b; Dunn et al, 1998; Hylan et al, 1998a, 1999a; Montejo et al, 1998).

\section{ADHERENCE TO DOSING REGIMEN}

Naturalistic data suggest that between $30 \%$ and $60 \%$ of patients do not take their medication as prescribed (Cramer, 1995; Demyttenaere, 1997). A 9-week RCT found that only $42.5 \%$ of patients took their antidepressant medication correctly (right dose, right time of day) $80 \%$ of the time (Demyttenaere et al, 1998); in a noncontrolled clinical setting, adherence might be expected to be even worse. Another study found that between $15 \%$ and $25 \%$ of patients had a gap of 2 weeks or more between their antidepressant prescriptions, which raises obvious questions about adherence to their medication (Hylan et al, 1998b). Many factors contribute to noncompliance with antidepressants, including the health care system, intrinsic pharmacological characteristics of the antidepressant (including adverse effects), and patient and prescriber behaviour (Demyttenaere, 1998).

A general perception exists that patients are more compliant with SSRIs than with TCAs. However, definitive evidence supporting this perception is lacking, although this may be attributable to the difficulty in studying issues of compliance (Demyttenaere, 1997). Simple methods of assessing compliance, such as pill counts and interrogation, are often inaccurate and generally overestimate adherence. More accurate methods of compliance assessment, such as electronic monitoring of medication bottle openings, are not simple to employ (Demyttenaere, 1997).

In a 9-week evaluation of compliance, assessed by use of a microelectromechanical system (MEMS) to monitor patients taking amitriptyline or fluoxetine (Demyttenaere et al, 1998), both patient groups were found to have poor compliance in general, with more than half the patients having treatment adherence of less than $50 \%$. No significant differences in treatment adherence were seen between groups. Interestingly, therapeutic effect was not correlated with compliance.

Another study has evaluated compliance in out-patients prescribed dothiepin or fluoxetine (Thompson et al, 2000) using these methods: pill count, patient report and MEMS monitoring. Similar compliance rates were found using pill count $(76 \%$ fluoxetine; $64 \%$ dothiepin) and patient report (79\% fluoxetine; $80 \%$ dothiepin) with both methods, suggesting that patients were generally compliant with their dosing regimen. However, when assessed using the MEMS, compliance was found to be less than that assessed by either pill count or patient report. The authors suggested that long windows (7 days or more) of total non-compliance over the course of the treatment period would be more damaging to treatment response than brief episodes spread out over the course of treatment, and defined a compliance index as a measure of this. Using the MEMS data to calculate the compliance index, adjusted mean compliance rates were significantly greater for fluoxetine-treated patients compared with dothiepin-treated patients $(70.0 \%$ fluoxetine; $55.8 \%$ dothiepin; $P=0.01$ ). Additionally, treatment response for both medications was significantly correlated with the compliance index $(P<0.001)$, but not with the $80 \%$ MEMS compliance measure. The discrepancy between the crude compliance measures (pill count and patient report) and the more rigorous method (MEMS) highlights some of the difficulties in studying this complex phenomenon.

One potential consequence of nonadherence to the prescribed regimen is the possibility of discontinuation symptoms, which have been described for both TCAs and SSRIs, with symptoms sometimes reported to occur after as little as one missed dose (Charney et al, 1982; Dilsaver \& Greden, 1984; Dilsaver et al, 1987; Schatzberg et al, 1997; Parker \& Blennerhassett, 1998). Comparative studies of individual SSRIs show that they have different propensities for discontinuation symptoms (Coupland et al, 1996; Lejoyeux et al, 1996; Price et al, 1996), with patients taking sertraline or paroxetine more likely to experience adverse events and a re-emergence of depressive symptoms on discontinuation than patients taking fluoxetine (Rosenbaum et al, 1998).

\section{DURATION OF THERAPY}

In clinical practice, only a small proportion of patients with depression experience adequate duration of therapy (Katon et al, 1992; MacDonald et al, 1996; Lepine et al, 1997; Donoghue, 1998b; Dunn et al, 1999), which is defined as 4-6 months beyond acute symptom resolution (World Health Organization Mental Health Collaborating Centers, 1989; Paykel \& Priest, 1992; Agency for Health Care Policy Research, 1993; British Association for Psychopharmacology, 1993; Reimherr et al, 1998). For example, in a population-based study from Scotland of over 400000 patients, only $32 \%$ of patients prescribed an antidepressant received more than 90 days of treatment (MacDonald et al, 1996). This duration of therapy is often required for acute symptom resolution, and does not meet the criteria for appropriate continuation therapy.

In primary care there is some limited evidence that TCA-treated patients discontinue treatment more rapidly than SSRItreated patients, with one study reporting that during a year's treatment, patients who began therapy on the TCA dothiepin received fewer prescriptions for the initially prescribed antidepressant than patients who began therapy on the SSRI fluoxetine (Treglia et al, 1999). In a retrospective Swedish study, patients who began treatment on an SSRI were more likely to have a longer initial therapy length (and possibly a reduced risk of recurrence) than patients who began therapy on a TCA. Subsequently, TCAtreated patients were more than twice as likely to have a recurrent episode of antidepressant treatment (at least 9 months between the end of the initial episode and restarting an antidepressant) than patients who began therapy on an SSRI (Isacsson et al, 1999).

Naturalistic studies of SSRIs have found that patients started on fluoxetine are more likely to complete a minimum course of therapy than patients started on 
other antidepressants (Katon et al, 1992; Sclar et al, 1994, 1995; Simon et al, 1996; Croghan et al, 1997; Montejo et al, 1998; Dunn et al, 1999; Hylan et al, 1999a; McCombs et al, 1999). In a large naturalistic study, patients initially prescribed sertraline were found to drop out of treatment at a significantly faster rate than patients prescribed fluoxetine (sertraline $v$. fluoxetine $P<0.001)$ or paroxetine (sertraline $v$. paroxetine $P<0.001$ ) (Donoghue, 1998b).

\section{LONGITUDINAL STUDIES OF TREATMENT PATTERN}

\section{TCA v. SSRI}

To overcome some of the limitations of RCTs and small naturalistic studies, a method was developed to conduct a longitudinal study in a large population. Using the DIN-Link database (Dunn et al, 1999), the study was designed to investigate the effect of the first antidepressant prescribed (by class, TCAs $v$. SSRIs) on the subsequent pattern of treatment in patients starting treatment for a new episode of depression. The main outcome measure was whether the patterns of treatment complied with national treatment guidelines (Paykel \& Priest, 1992).

Patients admitted to the study were initially prescribed one of the most commonly used TCAs (amitriptyline, dothiepin, imipramine or lofepramine) or SSRIs (fluoxetine, paroxetine or sertraline) for a new episode of depression (this was determined by a 6-month prior period during which time no treatment with any antidepressant had been prescribed). A population of 16204 patients entered the study, and the data were analysed on an intent-to-treat basis with all subsequent outcomes being assigned to the first antidepressant prescribed for each patient.

There may be several factors that influence prescribing patterns, so the data were subjected to logistic regression analysis to control for possible confounding variables and to determine the effect of the first antidepressant prescribed. The outcome measure was whether patients received antidepressant treatment at an effective dose for an adequate period. Factors that may have influenced the outcome, and which were controlled for in the analysis, included patient demographics (age and gender), region of the country, use of other health care resources, diagnosis of depression (the database permitted the use of five diagnostic labels for depression, including reactive depression and endogenous depression), comorbid physical illness and comorbid psychiatric conditions.

Of the 16204 patients entering the study, $62 \%$ were prescribed a TCA, with the remainder prescribed an SSRI. Even before controlling for factors that might have influenced outcome, patients initially prescribed an SSRI appeared much more likely to complete an effective course of therapy compared with those beginning treatment with a TCA. After controlling for potential confounding influences through logistic regression analysis, the odds of having an adequate dose of antidepressant and duration of treatment were over seven times higher for patients starting treatment with an SSRI (odds ratio 7.473, $P<0.001$ ). Patients prescribed TCAs failed to have their doses titrated to effective levels, with only $9 \%$ of patients having their dose increased. Even so, the majority of patients treated with a TCA discontinued treatment prematurely. Patients taking an SSRI always received an effective dose, but many discontinued treatment before an adequate duration of therapy had been achieved. The findings for the TCAs were skewed by the inclusion of lofepramine, which may be regarded as an atypical TCA, considerably less toxic in overdose (Henry et al, 1995) and generally considered to be better tolerated than older TCAs such as amitriptyline and imipramine. If lofepramine is excluded, the percentage of patients beginning treatment on TCAs who received adequate therapy falls to $2.8 \%$, and the odds ratio for SSRI-treated patients compared with TCA-treated patients receiving effective therapy increases to $17.1(P<0.001)$.

\section{SSRIs}

Differences in the use patterns of the SSRIs (in dosage and duration of treatment) in clinical practice are an unexpected finding. Since these compounds have many similarities (Leonard, 1993; Song et al, 1993; Anderson \& Tomenson, 1994; Finley, 1994; Henry et al, 1995), it seems possible that the differences in use patterns found in clinical practice may be due to external factors rather than to intrinsic properties of the compounds. To investigate this, data from the cohort prescribed SSRIs in the previous study were subjected to additional analysis (Dunn et al, 1998) to determine the effect of initiating treatment with fluoxetine, paroxetine or sertraline on the subsequent pattern of treatment. The main outcome measure was the likelihood of completing 6 months of continuous stable therapy on the initial antidepressant without switching to an alternative antidepressant, augmenting with a second antidepressant, or increasing the dose of the initial antidepressant. These alternatives were not considered to be inappropriate forms of treatment, but for the purposes of the study were regarded as signals of a delay in achieving the desired outcomes of antidepressant treatment, namely control of acute symptoms and subsequent recovery.

Patients admitted to the study were starting treatment with fluoxetine, paroxetine or sertraline for a new episode of depression (this was determined by a 6-month prior period during which time no treatment with any antidepressant had been prescribed). Of the 6150 patients entering the study, 3845 began treatment with fluoxetine, 1563 with paroxetine and 742 with sertraline. The data were analysed on an intent-to-treat basis with all subsequent outcomes being assigned to the first antidepressant prescribed for each patient. As in the previous study, observable factors that may have influenced the outcome were controlled for using logistic regression analysis.

Before adjustment for observable factors that might have influenced outcomes, patients beginning treatment with fluoxetine seemed most likely to achieve a minimum period of stable therapy. After controlling for potential confounding variables through logistic regression analysis, the odds of having a minimum period of stable therapy (relative to fluoxetine, which was assigned a value of 1$)$ were $0.44(P<0.001)$ for patients starting treatment with sertraline and $0.82(P<0.01)$ for patients starting treatment with paroxetine. However, since the study was not able to investigate outcomes, the clinical significance of these findings is unknown.

\section{DISCUSSION: WHY DO THESE OBSERVED DIFFERENCES OCCUR?}

Longitudinal studies of antidepressant use patterns confirm the findings of previous cross-sectional studies. Differences in use patterns are clearly demarcated by the class of antidepressant prescribed: patients who begin therapy on a TCA generally receive sub-therapeutic doses and discontinue the 
medication rapidly. In contrast, patients who begin therapy on an SSRI are more likely to have an adequate dose, although the duration of treatment is still shorter than that recommended in treatment guidelines. Within the SSRIs, differences also exist, although to a lesser degree. These differences are likely to affect the effectiveness of antidepressant treatment in the clinical setting.

It is possible that a single influence, a range of influences, or an interaction between them could cause these differences to occur. They could be due to pharmacological differences between the compounds. This seems plausible, as TCAs as a group have many similarities, as do the SSRIs. Controlled trials have shown that both groups of compounds have similar efficacy, so pharmacological differences are likely to be manifested as differences in tolerability, which could then be reflected in the prescribing patterns. Additionally, prescribing patterns are likely to be influenced by both the patient's behaviour and expectations of treatment, and the physician's behaviour and experience with treatments. Co-prescription of other medicines, concomitant physical or psychiatric illnesses, use of other health services and disease severity are also likely to influence prescribing patterns. Indeed, in the large longitudinal database studies described earlier (Dunn et al, 1998, 1999), the diagnostic criteria were not defined and disease severity was not assessed, both factors that might have influenced the outcomes observed. It has been suggested that many patients in primary care treated with TCAs at low doses actually suffer from mild, mixed anxiety-depression disorders and can be treated effectively with lower antidepressant doses than patients with major depression, although evidence to support this is lacking (Thompson \& Thompson, 1989a; Ali, 1998; Donoghue, 1998a). Similarly, a dose of dothiepin of $75 \mathrm{mg}$ per day was found to be effective in preventing recurrence of depression in elderly patients (Old Age Depression Interest Group, 1993), although doses prescribed for the elderly in clinical practice in primary care were found to be much lower than this (Donoghue et al, 1998). None the less, differences in use patterns, especially between TCAs and SSRIs, appear to be consistent across national boundaries, among patient populations who are likely to have very different health beliefs and expectations, in doctors who experience widely different training, and in systems of health care that deliver services in very different ways. The only constant influence appears to be biological: the differences in prescribing patterns occur as a result of pharmacological differences between the compounds.

The tolerability profile of an antidepressant can be expected to influence both adherence to dosing and overall duration of treatment (Kuzel et al, 1996; Demyttenaere et al, 1998). The SSRIs are generally considered to have tolerability advantages over TCAs, as they have a more benign adverse effect profile (Benfield \& Ward, 1986; Benfield et al, 1986; Dechant \& Clissold, 1991; Feighner et al, 1991; Edwards, 1992; Grimsley \& Jann, 1992; Murdoch \& McTavish, 1992; Edwards et al, 1994; Montgomery \& Kasper, 1998). In addition, the adverse effects associated with TCAs typically do not decrease in severity with continued therapy (Reimherr et al, 1988), whereas the side-effects of SSRIs generally tend to be transient (Reimherr et al, 1988; Zajecka et al, 1999) and more easily managed, for example by altering the time of administration (morning or evening) or taking the drug with food (Rickels \& Schweizer, 1990; Nemeroff, 1994; Kuzel et al, 1996). Of course, some patients on SSRIs do not experience symptom relief with continued therapy, and some sideeffects associated with SSRIs (such as sexual dysfunction) are not expected to be short-lived.

Meta-analyses of clinical trials have confirmed that more patients discontinue treatment because of adverse events on TCAs than on SSRIs (Montgomery et al, 1994; Anderson \& Tomenson, 1995; Montgomery \& Kasper, 1995; Geddes et al, 1999), although the differences in drop-out rates are modest. Other analyses have reported a higher incidence of adverse events with TCAs relative to fluoxetine, with significantly higher discontinuation rates for TCAs (Pande \& Sayler, 1993), and have found more adverse events reported with TCAs than with the SSRI citalopram (Bech \& Cialdella, 1992). Further evidence can be found in a study that accounted for both frequency and severity of side-effects, in which the sideeffect burden of amitriptyline was found to be substantially greater than that of sertraline (Reimherr et al, 1990), and in a controlled study where the rate of discontinuation due to adverse events was three times higher for the TCAs desipramine and imipramine than for fluoxetine (Simon et al, 1996).
The possibility that differences in prescribing patterns occur as a result of pharmacological differences is given additional weight by the logistic regression analyses conducted by Dunn et al (1998, 1999). This technique allows the potentially confounding influences of patient characteristics, region of the country, diagnosis of depression, concomitant physical and psychiatric illness, co-prescription of other medicines and the use of other health resources to be controlled for, with the results as reported above. It may be that there are still more influences at work of which we are as yet unaware, but on the evidence currently available, the most significant predictor of the subsequent pattern of treatment (which will, in its turn, determine the outcome in terms of response, recovery or likelihood of relapse) is the antidepressant with which treatment is initiated. The evidence clearly shows the advantage of initiating treatment with an SSRI.

Differences between the SSRIs are more difficult to explain, as pharmacological differences between them are small and none has been shown consistently to be better tolerated (Leonard, 1993; Finley, 1994). Clinical trials and pooled data from metaanalyses suggest that the SSRIs have more similarities than differences: similar efficacy, similar side-effects, similar tolerability and similar toxicity in overdose (Leonard, 1993; Song et al, 1993; Anderson \& Tomenson, 1994; Finley, 1994; Henry et al, 1995). However, despite these similarities, differences have been reported in their adverse effect profiles. Paroxetine has been associated with the highest incidence of antimuscarinic side-effects because of its affinity for cholinergic receptors (Gagiano, 1993; DeVane, 1995; Preskorn, 1995; Nelson, 1997), and citalopram has been associated with a higher degree of sedation because of its degree of binding to $\mathrm{H}_{1}$ receptors (Finley, 1994). Fluoxetine has been reported to produce an increase in the frequency of anxiety or anxiety-related symptoms including nervousness and agitation (DeVane, 1995; Fisher et al, 1995; Preskorn, 1995; Price et al, 1996; Nelson, 1997; MacKay et al, 1999), although other studies found fluoxetine to be similar to other SSRIs (Tignol, 1993; Patris et al, 1996; Fava et al, 1998). Weight loss and anorexia have been associated with fluoxetine (Tignol, 1993; Preskorn, 1995; Nelson, 1997; Michelson et al, 1999), while weight gain has been associated with paroxetine (Fava et al, 1998) and citalopram 
(Bouwer \& Harvey, 1996). Headache has been associated with fluoxetine (DeVane, 1995; Preskorn, 1995; Nelson, 1997; Ontiveros \& Garcia-Barriga, 1997). Based on data from clinical practice, the incidence of adverse events has been reported to be highest with fluvoxamine (MacKay et al, 1997; Arias et al, 1998). Gastrointestinal complaints, including nausea and vomiting, have been reported with fluvoxamine (Price et al, 1996; Arias et al, 1998), paroxetine (MacKay et al, 1999) and sertraline (DeVane, 1995; Fisher et al, 1995). Tremor has been reported with paroxetine (Price et al, 1996; MacKay et al, 1997, 1999), fluvoxamine (MacKay et al, 1997) and sertraline (Arias et al, 1998). Sexual dysfunction has been observed with all SSRIs, although reported to occur more frequently with paroxetine (Price et al, 1996; MacKay et al, 1997; Nelson, 1997; Fava et al, 1998; Rosen et al, 1999) and sertraline (DeVane, 1995; Fisher et al, 1995; Nelson, 1997).

There is, however, one property that separates fluoxetine from the other SSRIs. The pharmacokinetics of fluoxetine, with the very long half-life of its active metabolite norfluoxetine, suggest that until steadystate plasma levels are achieved (which may take up to 5 weeks), patients taking $20 \mathrm{mg}$ daily undergo very gradual incremental increases in plasma concentration. The incidence and discomfort of adverse effects is likely to be experienced more acutely in situations where treatment is initiated at a high dose, or where doses are increased rapidly. Thus, the long half-life of fluoxetine/norfluoxetine may confer greater tolerability, at least in the important early stages of treatment where the dropout rate is highest, which in turn results in more patients achieving a minimum period of stable therapy. The corollary to this, however, is that although adverse effects may be experienced less acutely, when they occur they will persist for longer than similar effects experienced with drugs with shorter half-lives. Similarly, problems may occur with drug interactions and on switching from fluoxetine to another antidepressant, when a long wash-out period may be required before another agent can be prescribed.

Because non-compliance and nonadherence to dosage in clinical practice is common, therapeutic coverage that is maintained despite missing doses may be beneficial. This benefit may be further enhanced if the potential for adverse effects when patients miss doses or abruptly discontinue therapy is reduced.

The data presented here show the disparity between the findings of controlled trials and those of observational studies. Controlled trials show no important differences in efficacy, and only modest tolerability advantages for the SSRIs compared with TCAs. Observational studies show that SSRIs appear to have important clinical advantages over TCAs, but the findings may be subject to bias. This creates a dilemma for practitioners over which results should take precedence. Although there seems to be a compelling case to abandon control of bias (controlled studies) in favour of generalisability (observational studies), a careful consideration of all the available evidence suggests that both sources of data are needed to make fully informed clinical decisions. Data from RCTs are essential to ensure that interventions for which efficacy has not been demonstrated are not applied in clinical practice, and observational studies provide information indicating which of a number of interventions of proven efficacy is most likely to deliver the desired outcome in a practice rather than a research setting.

\section{CONCLUSION}

Common behaviours in clinical practice include sub-therapeutic dosing, missing doses during therapy and inadequate lengths of therapy. These practices decrease the likelihood of acute symptoms improving and increase the likelihood of relapse or recurrent depressive episodes, demonstrating that there are significant opportunities for improvement in antidepressant prescribing. One possible opportunity is for individual prescribers to consistently select antidepressants that naturally maximise the potential for real-world efficacy.

Despite the proven efficacy of TCAs in controlled trials, patients treated with these antidepressants appear to have little hope of receiving adequate therapy. The findings of small naturalistic studies over many years - that patients prescribed TCAs are treated at doses that are not effective for the treatment of their depression - have been confirmed by large epidemiological studies which have revealed the pervasiveness of this practice, which seems to be independent of nationality or system of health care. More recent longitudinal analyses find that patients beginning treatment with a TCA start and remain on low doses, and still discontinue treatment prematurely.

In contrast, patients beginning treatment with an SSRI are at least seven times more likely to receive an adequate course of antidepressant therapy. Even so, a large proportion of patients drop out of treatment prematurely, leaving considerable scope for improvement.

These findings are restricted to the TCAs and SSRIs most commonly prescribed for the treatment of depression in primary care, and come largely from studies performed in the UK. On the basis of these data, SSRIs must be regarded as the gold standard for the treatment of depression in primary care. As newer antidepressants become used more widely, further studies will be required to evaluate the outcomes obtained from their use, and their potential to improve the management of depression.

\section{REFERENCES}

Agency for Health Care Policy Research (1993) Treatment of Major Depression, Vol. 2. Clinical Practice Guidelines, No. 5, Publication 93-0050. Rockville, MD: US Department of Health and Human Services.

Ali, I. M. (1998) Long-term treatment with antidepressants in primary care. Psychiatric Bulletin, 22 15-19.

Altamura, A. \& Percudani, M. (1993) The use of antidepressants for long-term treatment of recurrent depression: rationale, current methodologies, and future direction. Journal of Clinical Psychiatry, 54 (suppl. 8), 29-38.

Anderson, I. M. (1998) SSRIs versus tricyclic antidepressants in depressed inpatients: a meta-analysis of efficacy and tolerability. Depression and Anxiety, $\mathbf{7}$ (suppl. I), II-17

- (2000) Selective serotonin reuptake inhibitor versus tricyclic antidepressants: a meta-analysis of efficacy and tolerability. Journal of Affective Disorders, $\mathbf{5 8}$ 19-36.

\& Tomenson, B. M. (1994) The efficacy of selective serotonin reuptake inhibitors in depression: a metaanalysis of studies against tricyclic antidepressants. Journal of Psychopharmacology, 8, 238-249.

- \& - (1995) Treatment discontinuation rates with selective serotonin reuptake inhibitors compared with tricyclic antidepressants: a meta-analysis. British Medical Journal, 310, 1433-1438.

Anon. (1993) The treatment of depression in primary care. Effective Health Care, 5, I-12.

Arias, F., Padin, J. J., Gilaberte, I., et al (1998) Comparative efficacy and tolerability among different selective serotonin reuptake inhibitors and venlafaxine in a naturalistic setting. International journal of Psychiatry in Clinical Practice, 2. 255-260.

Beaumont, G., Baldwin, D. \& Lader, M. (1996) A criticism of the practice of prescribing subtherapeutic doses of antidepressants for the treatment of depression. Human Psychopharmacology, II, 283-29I. 
Bech, P. \& Cialdella, P. (1992) Citalopram in depression - meta-analysis of intended and unintended effects. International Clinical Psychopharmacology, 6 (suppl. 5), 45-54.

Benfield, P. \& Ward, A. (1986) Fluvoxamine: a review of its pharmacodynamic and pharmacokinetic properties, and therapeutic efficacy in depressive illness. Drugs, 32, 313-334.

—, Heel, R. C. \& Lewis, S. P. (1986) Fluoxetine. A review of its pharmacodynamic and pharmacokinetic properties, and therapeutic efficacy in depressive illness. Drugs, 32, 48I-508.

Berken, G. H., Weinstein, D. O. \& Stern, W. C. (1984) Weight gain: a side-effect of tricyclic antidepressants. Journal of Affective Disorders, 7, 133-138.

Bingefors, K., Isacson, D. \& von Knorring, L. (1997) Antidepressant dose patterns in Swedish clinical practice. International Clinical Psychopharmacology, 12 283-290.

Blashki, T. G., Mowbray, R. \& Davies, B. (197I) Controlled trial of amitriptyline in general practice. British Medical Journal, i, 133-138.

Bouwer, C. D. \& Harvey, B. H. (1996) Phasic craving for carbohydrate observed with citalopram. International Clinical Psychopharmacology, II, 273-278.

British Association for Psychopharmacology (1993) Guidelines for treating depressive illness with antidepressants: a statement from the British Association for Psychopharmacology. Journal of Psychopharmacology, 7, 19-23.

Canadian Coordinating Office (1998) A Clinical and Economic Evaluation of Selective Serotonin Reuptake Inhibitors in Major Depression. Ontario: Canadian Coordinating Office of Health Care Technology Assessment.

Charney, D. S., Heninger, G. R., Sternberg, D. E., et al (1982) Abrupt discontinuation of tricyclic antidepressant drugs: evidence for noradrenergic hyperactivity. British Journal of Psychiatry, 141, 377-386.

Coupland, N., Bell, C. J. \& Potokar, J. P. (1996) Serotonin reuptake inhibitor withdrawal. Journal of Clinical Psychopharmacology, 16, 356-362.

Cramer, J. A. (1995) Partial medication compliance: the enigma in poor medical outcomes. American Journal of Managed Care, I, 167-174.

Croghan, T. W., Lair, T. J., Engelhart, L., et al (1997)

Effect of antidepressant therapy on health care

utilization and costs in primary care. Psychiatric Services, 48, $1420-1426$.

Dechant, K. L. \& Clissold, S. P. (199I) Paroxetine. A review of its pharmacodynamic and pharmacokinetic properties, and therapeutic potential in depressive illness. Drugs, 4I, 225-253.

Demyttenaere, K. (1997) Compliance during treatment with antidepressants. Journal of Affective Disorders, 43, 27-39.

- (1998) Noncompliance with antidepressants: who' to blame? International Clinical Psychopharmacology, I3 (suppl. 2), S19-S25.

_ , Van Ganse, E., Gregoire, J., et al (1998) Compliance in depressed patients treated with fluoxetine and amitriptyline. International Clinical Psychopharmacology, 13, II-17.

DeVane, C. L. (1995) Comparative safety and tolerability of selective serotonin reuptake inhibitors. Human Psychopharmacology, 10 (suppl. 3), SI85-SI93.

Dilsaver, S. C. \& Greden, J. F. (1984) Antidepressant withdrawal phenomena. Biological Psychiatry, 19 237-256.
_ , _ \& Snider, R. M. (1987) Antidepressant withdrawal syndromes: phenomenology and physiopathology. International Clinical Psychopharmacology, 2, I-19.

Donoghue, J. M. (1998a) Sub-optimal use of tricyclic antidepressants in primary care (editorial). Acto Psychiatrica Scandinavica, 98, 429-431.

_ (1998b) Selective serotonin re-uptake inhibitor use in primary care: a five year naturalistic study. Clinical Drug Investigation, 16, 453-462.

_ \& Taylor, D. M. (2000) Suboptimal use of antidepressants in the treatment of depression. CNS Drugs, 13, 365-383.

_ , _ \& Tylee, A. (1996) The treatment of depression: prescribing patterns of antidepressants in primary care in the UK. British Journal of Psychiatry, $\mathbf{1 6 8}$ 164-168.

_ , _ \& Wildgust, H. (1996) Cross-sectional database analysis of antidepressant prescribing in general practice in the United Kingdom. British Medical Journal, 313, $861-862$

_, Katona, C. \& Tylee, A. (1998) The treatment of depression: antidepressant prescribing for elderly patients in primary care. Pharmaceutical Journal, 260, 500-502

Dunn, R. L., Donoghue, J. M., Ozminkowski, R. J. et al (1998) Selective serotonin reuptake inhibitor antidepressant prescribing in primary care in the United Kingdom. Primary Care Psychiatry, 8, |4I-148.

_, _ , _ et al (1999) Longitudinal patterns of antidepressant prescribing in primary care in the Unite Kingdom: a comparison to treatment guidelines. Journal of Psychopharmacology, 13, 136-143.

Dunner, D. \& Dunbar, G. C. (1992) Optimal dose regimen for paroxetine. Journal of Clinical Psychiatry, $\mathbf{5 3}$ (suppl. 2), 2I-26.

Edwards, J. G. (1992) Selective serotonin reuptake inhibitors. British Medical Journal, 304, I644-1646.

_ , Inman, W. H., Wilton, L., et al (1994) Prescription event monitoring of 10,401 patients treated with fluvoxamine. British Journal of Psychiatry, 164, 387-395.

Fabre, L. F., Abuzzahab, F. S., Amin, M., et al (1995) Sertraline safety and efficacy in major depression: a double-blind fixed-dose comparison with placebo. Biological Psychiatry, 38, 592-602.

Fava, M., Rosenbaum, J., Hoog, S., et al (1998) Fluoxetine versus sertraline and paroxetine in major depression: long-term changes in weight (abstract). European Neuropsychopharmacology, 8 (suppl. 2), S204.

Feighner, J. P., Gardner, E. A., Johnston, J. A., et al (199I) Double-blind comparison of bupropion and fluoxetine in depressed outpatients. Journal of Clinical Psychiatry, 52, 329-335.

Finley, P. R. (1994) Selective serotonin reuptake inhibitors: pharmacologic profiles and potential therapeutic distinctions. Annals of Pharmacotherapy, 28 1359-1369.

Fisher, S., Kent, T. A. \& Bryant, S. G. (1995) Postmarketing surveillance by patient self-monitoring: preliminary data for sertraline versus fluoxetine. Journal of Clinical Psychiatry, 56, 288-296.

Gagiano, C. A. (1993) A double-blind comparison of paroxetine and fluoxetine in patients with major depression. British Journal of Clinical Research, 4, 145-152.

Garland, E. J., Remick, R. A. \& Zis, A. P. (1988)

Weight gain with antidepressants and lithium. Journal of

Clinical Psychopharmacology, 8, 323-330.
Geddes, J. R., Freemantle, N., Mason, J., et al (1999) SSRls versus alternative antidepressants in depressive disorder. Cochrane Library, issue 4. Oxford: Update Software.

Gregor, K., Coons, S. J. \& MacDonald, R. (1994) Selective serotonin reuptake inhibitor dose titration in naturalistic setting. Clinical Therapeutics, 16, 306-315.

Grimsley, S. R. \& Jann, M.W. (1992) Paroxetine, sertraline, and fluvoxamine: new selective serotonin reuptake inhibitors. Clinical Pharmacy, II, 930-957.

Henry, J. A., Alexander, C. A. \& Sener, E. K. (1995) Relative mortality from overdose of antidepressants. British Medical Journal, 310, 221-224.

Hotopf, M., Lewis, G. \& Normand, C. (1996) Are SSRIs a cost-effective alternative to tricyclics? British Journal of Psychiatry, 168, 404-409.

Hylan, T. R., Dossenbach, M., Meneades, L., et al (1998a) Antidepressant use in the psychiatrist setting in Austria: a comparison of citalopram, fluoxetine, and paroxetine. Journal of Serotonin Research, 4 295-303.

_ , Dunn, R. L., Tepner, R., et al (1998b) Gaps in antidepressant prescribing in primary care in the United Kingdom. International Clinical Psychopharmacology, I3, 235-243.

_ , Crown, W. H., Meneades, L., et al (1999a) SSR antidepressant drug use patterns in the naturalistic setting. Medical Care, 37, AS36-AS44.

_ , Meneades, L., Crown, W. H., et al (1999b) SSR antidepressant use patterns and their relation to Clinical Global Impression scores: a naturalistic study. Journal of Affective Disorders, 52, III-II9.

Isacsson, G., Boethius, G., Henriksson, S., et al (1999) Selective serotonin reuptake inhibitors have broadened the utilisation of antidepressant treatment in accordance with recommendations. Findings from a Swedish prescription database. Journal of Affective Disorders, 53, I5-22.

Johnson, D. (1986) Noncompliance with antidepressant therapy - an underestimated problem. Internal Medicine, II, 14-17

Johnson, D. A. W. (1973) Treatment of depression in general practice. British Medical Journal, i, 18-20.

- (1981) Depression: treatment compliance in genera practice. Acta Psychiatrica Scandinavica, 63 (suppl. 290), 447-453.

Katon, W., von Korff, M., Lin, E., et al (1992) Adequacy and duration of antidepressant treatment in primary care. Medical Care, 30, 67-76.

Katzelnick, D., Kobak, K. A., Jefferson, J., et al (1996) Prescribing patterns of antidepressant medications for depression in an HMO. Formulary, 31, 374-388.

Keller, M. B., Klerman, G. L., Lavori, P. W., et al (1982) Treatment received by depressed patients. Journal of the American Medical Association, 248 1848-1855.

Kotlin, J., Post, R. M. \& Goodwin, F. K. (1973) Drug treatment of depressed patients referred for hospitalisation. American Journal of Psychiatry, 130 |139-||4|.

Kuzel, R. J., De Wester, J. N. \& Richardson, F. (1996) Treating comorbid depression and anxiety. Journal of Family Practice, 43 (suppl. 6), S45-S53.

Lejoyeux, M., Ades, J., Mourad, I., et al (1996) Antidepressant withdrawal syndrome recognition, prevention and management. CNS Drugs, $\mathbf{5}$, 278-292. 
Leonard, B. E. (1993) The comparative pharmacology of new antidepressants. Journal of Clinical Psychiatry, $\mathbf{I 5}$ (suppl. 3), 54-58.

Lepine, J. P., Gastpar, M., Mendlewicz, J., et al (1997) Depression in the community: the first pan-European study DEPRES (Depression Research in European Society). International Clinical Psychopharmacology, I2, 19-29.

\section{Li, J., Claxton, A. J. \& McKendrick, J. (1998)} Antidepressant prescribing patterns and the risk of relapse and recurrence of depression in a primary care cohort in the United Kingdom. European

Neuropsychopharmacology, 8 (suppl. 2), SI66.

MacDonald, T. M., McMahon, A. D., Reid, I. C., et a (1996) Antidepressant drug use in primary care: a record linkage study in Tayside, Scotland. British Medical Journal, 313, 860-86।.

MacKay, F. R., Dunn, N. R., Wilton, L. V., et al (1997) A comparison of fluvoxamine, fluoxetine, sertraline and paroxetine examined by observational cohort studies. Pharmacoepidemiology and Drug Safety, 6, 235-246.

_, _, Martin, R. M., et al (1999) Newer antidepressants: a comparison of tolerability in general practice. British Journal of General Practice, 49, 892-896.

Maj, M., Veltro, F., Pirozzi, R., et al (1992) Pattern of recurrence of illness after recovery from an episode of major depression: a prospective study. American journal of Psychiatry, 149, 795-800.

McCombs, J. S., Nichol, M. B. \& Stimmel, G. L. (1999) The role of SSRI antidepressants for treating depressed patients in the California Medicaid (Medi-Cal) program. Value in Health, 2, 269-280.

Melfi, C. A., Chawla, A. J., Croghan, T.W., et al (1998) Effect of adherence to depression treatment guidelines on relapse and recurrence. Archives of General Psychiatry, 55, $1128-1132$

Michelson, D., Amsterdam, J. D., Quitkin, F. M., et al (1999) Changes in weight during a I-year trial of fluoxetine. American Journal of Psychiatry, 156, II70-1176.

Mintz, J., Mintz, L. I., Arruda, M. J., et al (1992) Treatments of depression and the functional capacity to work. Archives of General Psychiatry, 49, 76I-768.

Montejo, A. L., Gilaberte, I., Fombellida, C., et al (1998) Pattern of usage of new antidepressants in clinic practice. Actas Luso-Españolas de Neurologia, Psiquiatria y Ciencias Afines, 26, 75-82.

Montgomery, S. A. \& Kasper, S. (1995) Comparison of compliance between serotonin reuptake inhibitors and tricyclic antidepressants: a meta-analysis. International Clinical Psychopharmacology, 9 (suppl. 4), $33-40$

— \& _ (1998) Side effects, dropouts from treatment and cost consequences. International Clinical Psychopharmacology, 13 (suppl. 2), SI-S5.

_, Rasmussen, J. G., Lyby, K., et al (1992) Dose response relationship of citalopram $20 \mathrm{mg}$, citalopram $40 \mathrm{mg}$ and placebo in the treatment of moderate and severe depression. International Clinical Psychopharmacology, 6 (suppl. 5), 65-70.

_, Henry, J., McDonald, G., et al (1994) Selective serotonin reuptake inhibitors: meta-analysis of discontinuation rates. International Clinical Psychopharmacology, 9, 47-53.

Munizza, C., Tibaldi, G., Bollini, P., et al (1995) Prescription pattern of antidepressants in out-patient psychiatric practice. Psychological Medicine, 25, 77I-778.

Murdoch, D. \& McTavish, D. (1992) Sertraline. A review of its pharmacodynamic and pharmacokinetic properties, and therapeutic potential in depression and obsessive-compulsive disorder. Drugs, 44, 604-624.

Navarro, R., Valler, W. E., Spangler, M., et al (1995) Antidepressant utilization in managed care: an evaluation of SSRI use in two HMO settings. Medical Interface, 8, I14-123.

Nelson, J. C. (1997) Safety and tolerability of the new antidepressants. Journal of Clinical Psychiatry, 58 (suppl. 6), 26-31.

Nemeroff, C. B. (1994) Evolutionary trends in the pharmacotherapeutic management of depression. Journal of Clinical Psychiatry, 55 (suppl. 12), 3-15.

Old Age Depression Interest Group (1993) How long should the elderly take antidepressants? A double-blind placebo-controlled study of continuation/prophylaxis therapy with dothiepin. British Journal of Psychiatry, 162 175-182.

Ontiveros, A. \& Garcia-Barriga, C. (1997) A doubleblind, comparative study of paroxetine and fluoxetine in out-patients with depression. British Journal of Clinical Research, 8, 23-32.

Pande, A. C. \& Sayler, M. E. (1993) Adverse events and treatment discontinuations in fluoxetine clinical trials. International Clinical Psychopharmacology, 8 267-269.

Parker, G. \& Blennerhassett, J. (1998) Withdrawal reactions associated with venlafaxine. Australian and New Zealand journal of Psychiatry, 32, 291-294.

Patris, M., Bouchard, J. M., Bougerol, T., et al (1996) Citalopram versus fluoxetine: a double-blind controlled, multicentre, phase III trial in patients with unipolar major depression treated in general practice. International Clinical Psychopharmacology, II, 129-136.

Paykel, E. S. \& Priest, R. G. (1992) Recognition and management of depression in general practice: consensus statement. British Medical Journal, 305 1198-1202.

\section{_, Mueller, P. S. \& De la Vergne, P. M. (1973)} Amitriptyline, weight gain and carbohydrate craving: a side effect. British Journal of Psychiatry, 123, 50I-507.

Phillips, S., Brent, J., Kulig, K., et al (1997) Fluoxetine versus tricyclic antidepressants: a prospective multicenter study of antidepressant drug overdoses. Journal of Emergency Medicine, I5, 439-445.

Preskorn, S. H. (1995) Comparison of the tolerability of bupropion, fluoxetine, imipramine, nefazodone, paroxetine, sertraline, and venlafaxine. Journal of Clinical Psychiatry, 56 (suppl. 6), 12-21.

Price, J. S., Waller, P. C., Wood, S. M., et al (1996) A comparison of the post-marketing safety of four selective serotonin re-uptake inhibitors including the investigation of symptoms occurring on withdrawal. British Journal of Clinical Pharmacology, 42, 757-763.

Prien, R. F. \& Kupfer, D. J. (1986) Continuation drug therapy for major depressive episodes: how long should it be maintained? American Journal of Psychiatry, 143 18-23.

Reimherr, F.W., Byerley, W. F., Ward, M. F., et al (1988) Sertraline, a selective inhibitor of serotonin uptake, for the treatment of outpatients with major depressive disorder. Psychopharmacology Bulletin, 24, 200-205.

\section{_ , Chouinard, G., Cohn, C. K., et al (1990)}

Antidepressant efficacy of sertraline: a double-blind, placebo- and amitriptyline-controlled multicenter comparison study in outpatients with major depression. Journal of Clinical Psychiatry, 5I (suppl B), 18-27.

_ , Amsterdam, J. D., Quitkin, F. M., et al (1998) Optimal length of continuation therapy in depression: a prospective assessment during long-term fluoxetine treatment. American Journal of Psychiatry, 155 1247-1253.

Rickels, K. \& Schweizer, E. (1990) Clinical overview of serotonin reuptake inhibitors. Journal of Clinical Psychiatry, 5I (12, suppl. B), 9-12.

Rosen, R. C., Lane, R. M. \& Menza, M. (1999) Effects of SSRIs on sexual function: a critical review. Journal of Clinical Psychopharmacology, 19, 67-85.

Rosenbaum, J., Fava, M., Hoog, S. L., et al (1998) Selective serotonin reuptake inhibitor discontinuation syndrome: a randomized clinical trial. Biological Psychiatry, 44, 77-87.

Rosholm, J. U., Hallas, J. \& Gram, L. F. (1993)

Outpatient utilization of antidepressants: a prescription database analysis. Journal of Affective Disorders, 27, $21-28$.

Rudolph, R. L., Entsuah, R. \& Chitra, R. (1998) A meta-analysis of the effects of venlafaxine on anxiety associated with depression. Journal of Clinical Psychiatry 59, $116-122$.

Schatzberg, A. F., Haddad, P., Kaplan, E. M., et al (1997) Serotonin reuptake inhibitor discontinuation syndrome: a hypothetical definition. Discontinuation Consensus Panel. Journal of Clinical Psychiatry, 58 (suppl. 7), 5-10.

Sclar, D. A., Robison, L. M., Skaer, T. L., et al (1994) Antidepressant pharmacotherapy: economic outcomes in a health maintenance organization. Clinical Therapeutics, 16, 715-730.

, et al (1995) Antidepressant pharmacotherapy: economic evaluation of fluoxetine, paroxetine and sertraline in a health maintenance organization. Journal of International Medical Research, 23, 395-412

Scott, J. (1988) Chronic depression. British Journal of Psychiatry, I53, 287-297.

Simon, G. E., Wagner, E. \& Von Korff, M. (1995) Cost-effectiveness comparisons using real-world randomized trials: the case of new antidepressant drugs. Journal of Clinical Epidemiology, $\mathbf{4 8}$ 363-373.

_ , Von Korff, M., Heiligenstein, J. H., et al (1996)

Initial antidepressant choice in primary care: effectiveness and cost of fluoxetine vs. tricyclic antidepressants. Journal of the American Medical Association, 275, 1897-1902.

Song, F., Freemantle, N., Sheldon, T. A., et al (1993) Selective serotonin reuptake inhibitors: meta-analysis of efficacy and acceptability. British Medical Journal, 306, 683-687.

Sturm, R. \& Wells, K. B. (1995) How can care for depression become more cost-effective? Journal of the American Medical Association, 273, 51-58.

Thompson, C. \& Thompson, C. M. (1989a) The prescription of antidepressants in general practice. I: A critical review. Human Psychopharmacology, 4, 91-107.

_ \& _ (1989b) Prescribing of antidepressants in general practice. II: A placebo controlled trial of low dose dothiepin. Human Psychopharmacology, 4, I9I-204.

_, Peveler, R. C., Stephenson, D., et al (2000) Compliance with antidepressant medication in the treatment of major depressive disorder in primary care: a randomized comparison of fluoxetine and a tricyclic antidepressant. American Journal of Psychiatry, $\mathbf{I 5 7}$ 338-343.

Tignol, J. (1993) A double-blind, randomized, fluoxetine-controlled, multicenter study of paroxetine in the treatment of depression. Journal of Clinical Psychopharmacology, 13 (6, suppl. 2), I8S-22S. 
Treglia, M., Neslusan, C. A. \& Dunn, R. L. (1999)

Fluoxetine and dothiepin therapy in primary care and health resource utilization: evidence from the United Kingdom. International Journal of Psychiatry in Clinical Practice, 3, 23-30

Truter, I. \& Kotze, T. J. (1996) An investigation into the prescribing patterns of selective serotonin reuptake inhibitors in South Africa. Journal of Clinical Pharmacy and Therapeutics, 2I, 237-242.

Wernicke, J. F., Bosomworth, J. C. \& Ashbrook, E. (1989) Fluoxetine at $20 \mathrm{mg}$ per day: the recommended and therapeutic dose in the treatment of depression. International Clinical Psychopharmacology, 4 (suppl. I), 63-67.

JOHN DONOGHUE, MRPharmS, School of Pharmacy and Chemistry, Liverpool John Moores University, Liverpool, UK; TIMOTHY R. HYLAN, PhD, CNS Medical Service Liaison Team, Pfizer, Inc., USA

Correspondence: Mr John Donoghue, PCS Health, 4 Wrenfield Grove, Liverpool LI7 9QD, UK. Tel: +44 I5I 726 1860; e-mail: john@donoghue.u-net.com

World Health Organization Mental Health Collaborating Centers (1989) Pharmacotherapy of depressive disorders: WHO consensus statement. Journal of Affective Disorders, 17, 197-198.
Zajecka, J., Amsterdam, J. D., Quitkin, F. M., et al (1999) Changes in adverse events reported by patients during six months of fluoxetine therapy. Journal of Clinical Psychiatry, 60, 389-394. 